\title{
Palmengarten 2008
}

\author{
Andrea MÜller \& MatThias Jenny
}

Januar: Zartrote Blütenträume: Für den Palmengarten sind die milden Temperaturen im Januar ein Geschenk - noch nie konnte die Kamelienausstellung so früh eröffnet werden. 200 Gäste geraten beim Anblick der zarten Blütenpracht ins Schwärmen und Fachsimpeln. Passend zur fernöstlichen Ausstellung wird japanischer grüner Tee gereicht. Überschattet werden die Vorbereitungen zur Ausstellung durch den plötzlichen Tod des Ausstellungskoordinators PETER GUTMANN, der stets hinter den Kulissen, aber mit vollem Einsatz über viele Jahre die Ausstellungen im Palmengarten mitgeprägt hat.

$\mathrm{Zu}$ viel Licht im Palmengarten: Eine kleine Partei argwöhnt, dass im Palmengarten zu viel Strom für die Beleuchtung verbraucht wird. Der Palmengarten sei dem Umweltdezernat zugeordnet und habe daher eine Vorbildfunktion für die städtische Energie- und Klimabilanz. Dass der Palmengarten für die Beheizung der Gewächshäuser viel Energie verbraucht, ist eine Tatsache. Tatsache ist aber auch, dass der Palmengarten seit Jahren einen Energiebeauftragten hat, der zum Beispiel dafür sorgt, dass tagsüber keine Lichter unnötig brennen und dass in allen Lampen entlang der Wege Energiesparlampen eingesetzt werden.

Oh là là, Francfort: Der französische Generalkonsul HENRI REYNAUD bekennt sich in einem Zeitungsinterview zum Palmengarten: „Dass Frankfurt so grün ist, hat mich positiv überrascht. Meine Freunde haben damals zu mir gesagt: ,Oh là là, Francfort, da wirst du keine Bäume sehen.' Jetzt spaziere ich täglich durch den Palmengarten - der liegt genau zwischen dem Konsulat und meiner Wohnung im Westend. Sogar nachts darf ich in die Anlage, denn ich habe ein Jahresabonnement!“

Grüne Juwelen: Die Frankfurter Universitätsbibliothek JOHANN CHRISTIAN SENCKENBERG ist um einen bedeutenden Buchbestand reicher: Knapp 1000 historische Bände aus der ehemaligen Königlichen Gartenbibliothek Herrenhausen in Hannover werden der Öffentlichkeit vorgestellt. Mit einem Investitionsvolumen von 1,2 Millionen Euro handelt es sich um eine der größten Erwerbungen in der Geschichte der Universitätsbibliothek. Der Bestand umfasst überwiegend Drucke zum Thema Botanik und Gartenbau aus dem 16. bis 19. Jahrhundert. Der Palmengarten freut sich mit der Universität über die botanische Bereicherung der Bibliothek, insbesondere weil die Herrenhäuser Bibliothek schon 2005 im Palmengarten präsentiert und so einer breiten Öffentlichkeit zugänglich gemacht wurde.

Februar: Nachts im Dschungel: „Wir garantieren Ihnen, dass Sie heute Ihr Fett wegkriegen.“ Unter dem Motto „Voll Fett“ geht es bei dem nächtlichen Rundgang in diesem Jahr um Fette, Öle und Wachse aus dem Pflanzenreich. Etwa um den Topfuntersetzer aus Wacholderscheiben, der zu duften beginnt, sobald man eine heiße Teekanne draufsetzt. „Die Pflanzen haben ätherische Öle, um Insekten anzulocken, sich in heißen Gefilden zu kühlen oder sich mit strengen Aromen vor Fraß zu schützen“, erklärt HILKE STEINECKE der kleinen Menschenkarawane, die durchs dunkle Tropicarium zieht.

HaiYan Li, der Chinesische Generalkonsul begrüßt zur Feier des chinesischen Neujahrsfestes über 200 Prominente in der neuen Residenz an der Stresemannallee. Er kündigt auch gemeinsam mit der Oberbürgermeisterin PETRA RоTH die Frankfurter Tage in Guangzhou an. An den Präsentationen in der chinesischen Partnerstadt Frankfurts ist der Palmengarten prominent vertreten durch die ins Chinesische übertragene Ausstellung „GOETHE und die Pflanzenwelt" und die Anlage eines Frankfurter Gartens.

März: Entstaubt und aufgepeppt: Die „Frischzellenkur “ für die Gartenausstellung - das 2007 erstmals eingeführte neue Konzept - zeigt Wirkung. Bei Veilchenblüten-Espresso und Rosenmus ist „Garten 2008“ ein Fest für die Sinne. Ein besonderer Akzent der Ausstellung liegt in die- 

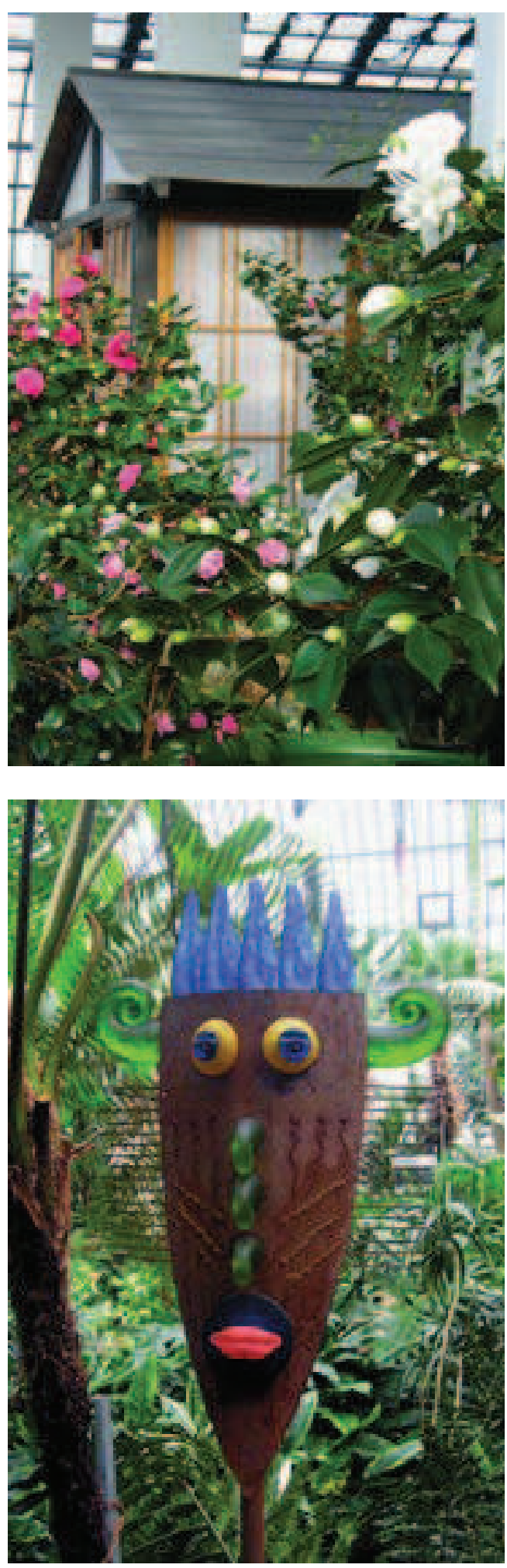

sem Jahr auf der Gartenkunst. Hierbei sind Kunstobjekte aus Stahl, Kupfer, Sand- oder Natursteinen von verschiedenen Künstlern unter freiem Himmel zu sehen. Im Palmenhaus sind Objekte eines Glasbläsers zu bestaunen, es gibt eine Fotoschau und Buchvorstellung. Der Gezeiten-Klanggarten, für den eigens ein ViermastZirkuszelt auf der Liegewiese aufgebaut wird, ist dieses Jahr eine besondere Attraktion. Auch der Stand von BioFrankfurt bildet einen Schwerpunkt und macht die „(G)artenvielfalt der Rhein-Main-Region“ für die Besucher erlebbar. Insgesamt sind rund 90 Aussteller auf einer Fläche von 4000 qm vom 13. bis 16. März zu sehen. Besonders erfreulich: es blüht bereits überall, Krokusse, Stiefmütterchen, Forsythien. Rund 80000 Blüten sind zu bewundern, selbst die Mandeln blühen, zum ersten Mal während der Gartenausstellung. Trotz Niesel- und Regenwetter kommen rund 25000 Besucher zu „Garten 2008 “.

Kälteeinbruch an Ostern: Den plötzlichen Kälteeinbruch über Ostern überstehen viele Blüten leider nicht. Die Vegetation ist aufgrund des recht milden Winters einige Wochen weiter als sonst, weshalb die Kälte besonders viel Schaden verursacht. Die Blüten der Magnolien und Kamelien, die sonst um diese Jahreszeit den Frühling ankündigen, sind fast alle erfroren.

Der Schneesturm und die Kälte können jedoch dem fröhlichen Ostereiersuchen im Palmengarten nichts anhaben. Dank der Galerien kann dies glücklicherweise im Warmen und Trockenen stattfinden, worüber sich rund 4000 Besucher freuen.

Tierische Neuzugänge: Niemand weiß, wie sie in den Palmengarten gekommen sind, jedoch stolziert neuerdings der Südchinesische Silberfasan mit seinen drei Weibchen durch unsere farbenprächtigen Wiesen. Somit haben die Besucher eine zusätzliche tierische Attraktion im Palmengarten.

Abb 1 (oben): Teehaus in der Kamelien-Ausstellung.

Abb. 2 (unten): Lustige Figuren schmücken während der Gartenmesse 2008 das Palmenhaus. 


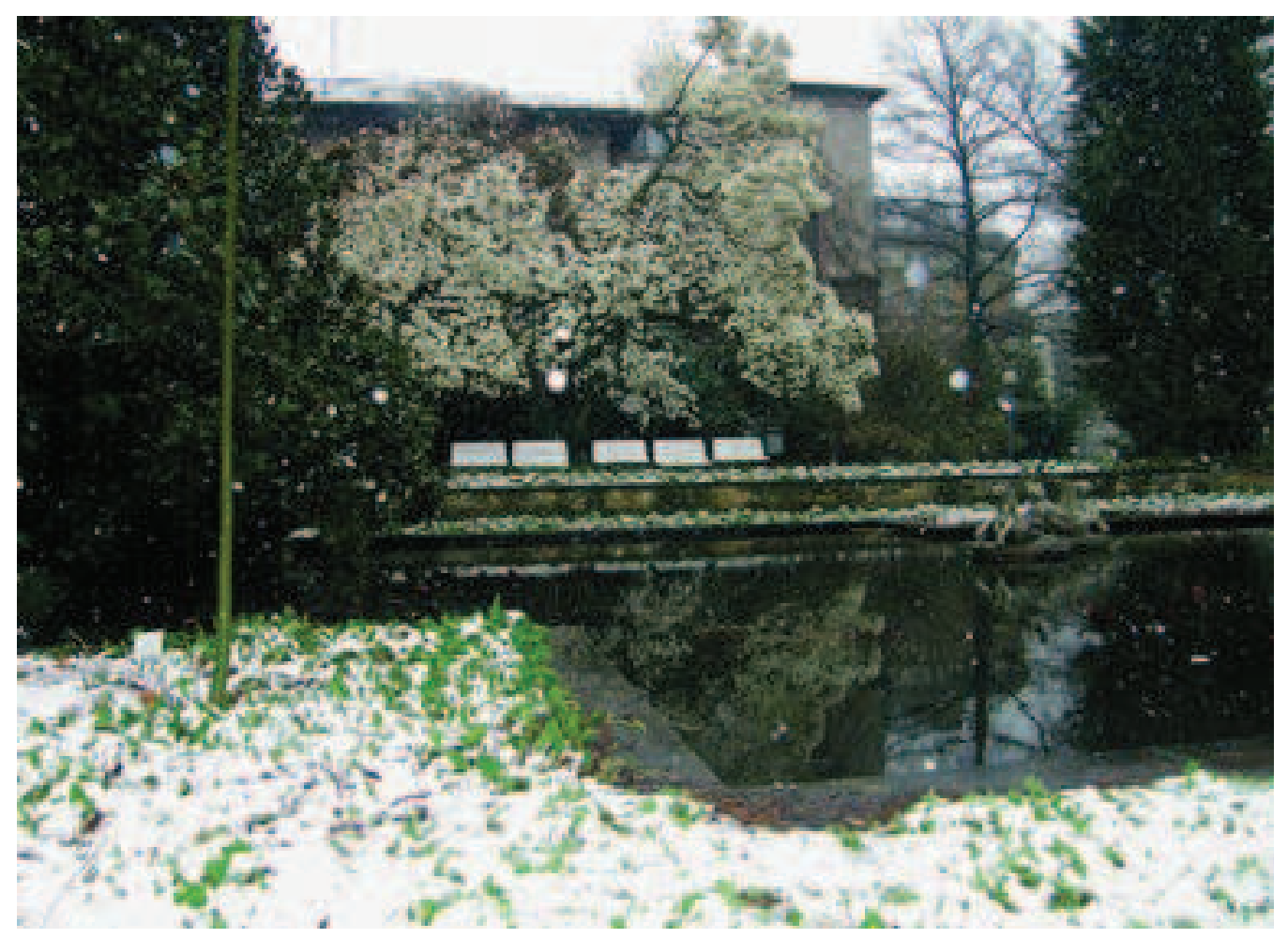

Sommerliche Temperaturen: Am letzten Märzwochenende werden sommerliche Temperaturen verzeichnet und im Palmengarten herrscht entsprechend gute Stimmung. Es gibt bereits Mohnblumen, Kamelien und insbesondere Azaleen zu bewundern. Jedoch ist im Palmengarten noch nicht alles auf Sommer eingestellt: Zum Ärger einiger Besucher hat beispielsweise der Minigolfplatz noch zu, da die Bahnen generalüberholt werden müssen.

April: Palmengarten lässt in China Blumen sprechen: Die Reise der Frankfurter Delegation um Oberbürgermeisterin PETRA ROTH zum 20-jährigen Jubiläum der Partnerschaft mit der chinesischen Stadt Guangzhou ist wegen der Tibet-Unruhen umstritten. Auf kultureller Ebene sind die Verbindungen jedoch nach wie vor eng und der Palmengarten lässt Blumen sprechen: Der Rosengar-

Abb. 3: Seltener Anblick: vom Schnee überraschte blühende Yulan-Magnolie. ten, den Frankfurt zur 2200-Jahrfeier Guangzhous im Jahre 1996 schenkte, wird derzeit von Mitarbeitern des Palmengartens saniert. Gärtnermeister ROLAND RUDOLPH reist mit 350 Steinfurther Rosen im Gepäck nach China, um die dortigen altersschwachen und unter dem subtropischen Klima leidenden Rosen im LiU-HuA-Hu-Park zu ersetzen. Parallel dazu wird drei Wochen lang die Palmengarten-Ausstellung über GOETHE und die Pflanzenwelt gezeigt, die am 9. April zusammen mit dem neuen Rosengarten von Oberbürgermeisterin PETRA ROTH in China eröffnet wird.

Empfindsame Schöne: Orchideen sorgen im Palmengarten vom 18. bis 27. April für wundervolle Farbenpracht und Formenvielfalt. „So üppig war die Orchideen-Ausstellung noch nie“, findet nicht nur Stadtverordnetenvorsteher KARLHEINZ BÜHRMANN. Studierende der Hochschule für Gestaltung Offenbach zeigen in der Galerie Zeichnungen, die im Palmengarten entstanden sind.

Es wird im Grünen unterrichtet: Das Projekt „Kinder erleben Natur“ zeigt, wie vielfältig Tiere, Pflanzen und Lebensräume sind. Vier Frankfurter Institutionen - Stadtwaldhaus, Zoo, 


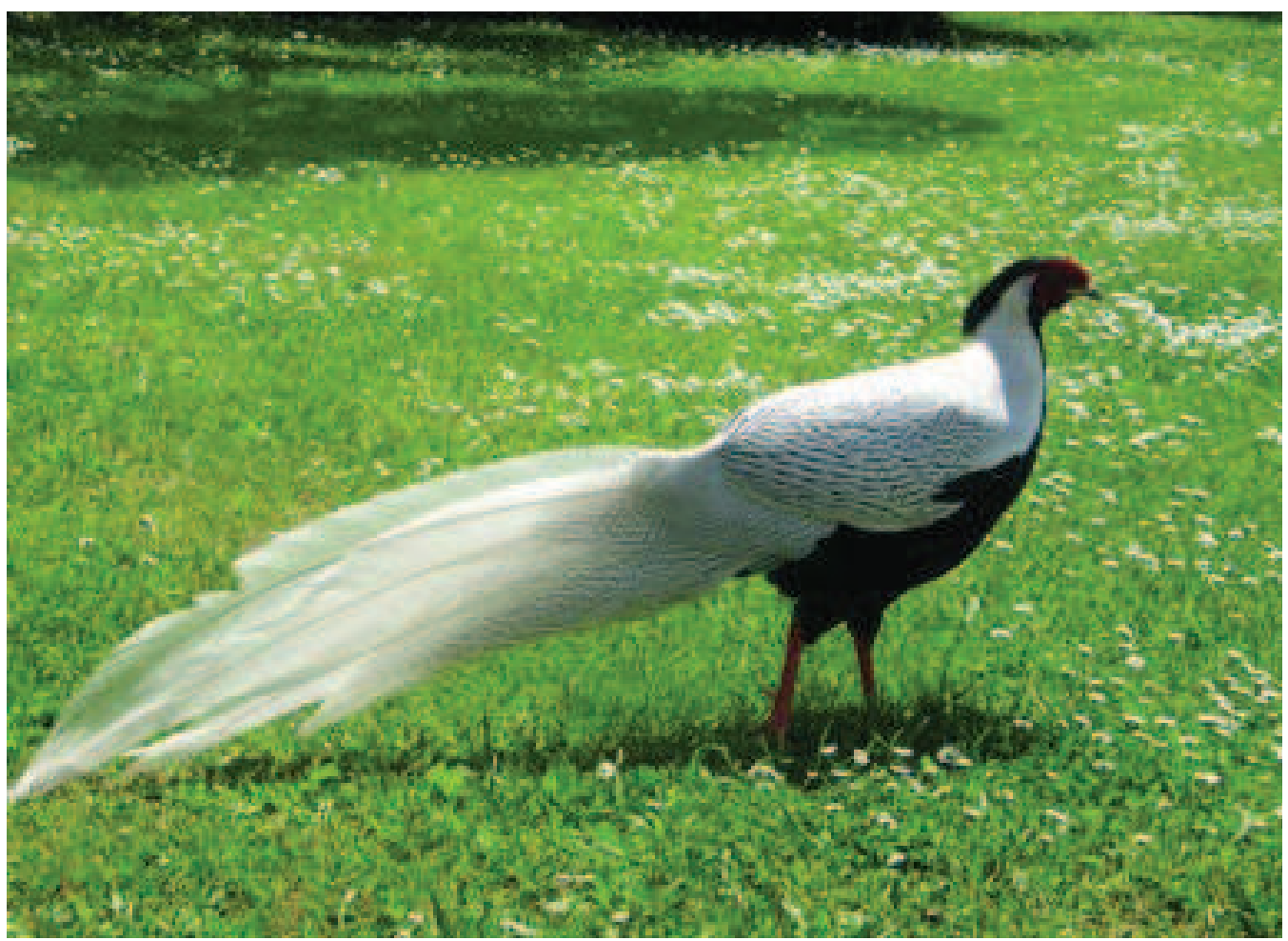

Senckenbergmuseum und Palmengarten - haben sich die Aufgabe gestellt, die Biodiversität für Schulkinder in einem aufeinander abgestimmten Programm darzustellen. Der Palmengarten zeigt im Rahmen dieser Aktion tropische Nutzpflanzen wie Kakao, Kokospalme, Ananas oder Zuckerrohr.

Mai: Maien-Jazz im Palmengarten: Die EZB feiert ihr zehnjähriges Bestehen mit hochkarätigem Musikprogramm im Palmengarten. Am Musikpavillon treffen am 20. Mai die Jazz-Fans ein, um dem Christof LaUer Trio zusammen mit Nils Landgren und Wolfgang Pusching zu lauschen. Mit dieser Besetzung bringt die EZB einige der wichtigsten Jazzmusiker Europas in den Palmengarten.

Pfingsten wird bunt: Das schöne Wetter hat im Palmengarten zahlreiche Blüten hervorgelockt, und da dieses Jahr Pfingsten so früh liegt, blühen die Pfingstrosen passend zu den Feiertagen. Wunderschön ist auch der Rhododendrongarten am großen Weiher, wo sich bunte Farben im Wasser spiegeln.
„Kann Natur privatisiert werden?" Mit dieser Frage beschäftigt sich am 18. Mai eine Diskussion im Siesmayersaal. Die Veranstaltung ist Teil der Weltkonferenz zur biologischen Vielfalt, die in Bonn abgehalten wird.

Braunes Gold Kakao: „Biologische Vielfalt erleben" heißt das Motto der Aktionswoche, die vom 22. bis 31. Mai in verschiedenen Naturschutz-, Forschungs- und Bildungseinrichtungen veranstaltet wird. Im Palmengarten kann man während dieser Woche an Führungen für die ganze Familie zum Thema „Kakao - vom Kakaobaum zur Schokocreme" teilnehmen.

Juni: „Die Fledermaus“ im Pagageno-Musiktheater: Mit der STRAUSS-Operette feiert das Papageno seinen zehnten Geburtstag. Junge Opernsängerinnen und -sänger stellen hierbei ihr Können unter Beweis. Theaterleiter HaNS-

Abb. 4: Silberfasan. 


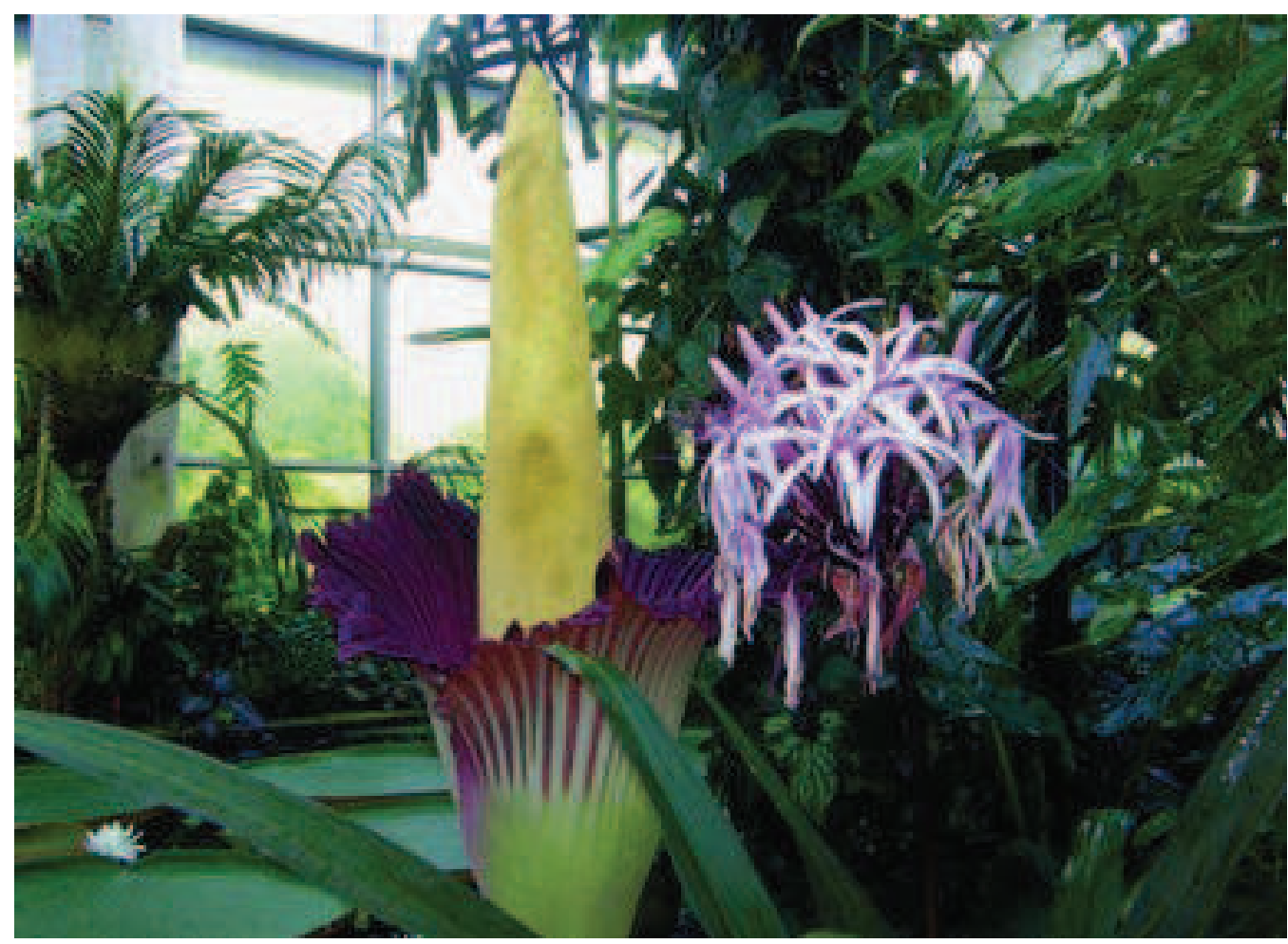

Dieter Maienschein legt großen Wert auf die Förderung junger Talente und bietet mit dem Papageno eine Plattform für Nachwuchskünstler. Zur Sonne, zur Freiheit: Der Palmengarten hat eine der größten Agaven-Sammlungen Europas, und zwei Agaven bilden im Juni einen riesigen Blütenstand. Die eine arbeitet sich bis in eine Höhe von 5 Metern vor, so dass Mitarbeiter eine Scheibe aus dem Glasdach des Gewächshauses herausnehmen müssen. Der Blütenstand ragt nun kerzengerade drei Meter über das Dach hinaus. Im Gegensatz zu Pflanzen, die jährlich blühen, brauchen Agaven oft viele Jahrzehnte, bis sie dann alle Energie in den Blütenstand stecken.

Zehntausende Rosen blühen beim Rosenund Lichterfest: Die Voraussetzungen für das diesjährige Rosen- und Lichterfest sind aufgrund der Fußball-Europameisterschaft und des kalten,

Abb. 5: Begeistert immer wieder: eine blühende Titanenwurz, diesmal sehr schön zusammen mit der größten Seerose. regnerischen Wetters nicht die besten. Dennoch erfreuen sich rund 14000 Besucher an der Pracht der Rosen sowie dem Lichterfest. Der neue Rosengarten rund um das Haus Rosenbrunn verzaubert wieder mit bunter Blütenfülle, auch das in diesem Frühjahr fertiggestellte DuftRosenbeet sowie der neu gestaltete südliche Bereich begeistern die Besucher. 4000 neue Rosenstöcke in 44 Sorten gibt es zu bestaunen. Es ist überhaupt ein Wunder, dass die Rosen im ersten Jahr schon so schön blühen.

Rätsel um schwarze Palmengarten-Schwäne: Über die verschwundenen Eier der schwarzen Schwäne grübeln Mitarbeiter und Besucher des Palmengartens. Fünf Eier hat das SchwanenPärchen auf der Insel des Weihers ins Nest gelegt und die Freude über möglichen Nachwuchs ist groß. Doch plötzlich sind alle Eier spurlos verschwunden. Nach wochenlangem erfolglosem Brüten haben sie die vermutlich unbefruchteten Eier verstoßen.

Palmengarten-Spielplatz wird renoviert: Nach einer Routineüberprüfung der Spielplätze durch das Grünflächenamt wurde aus Sicherheitsgrün- 


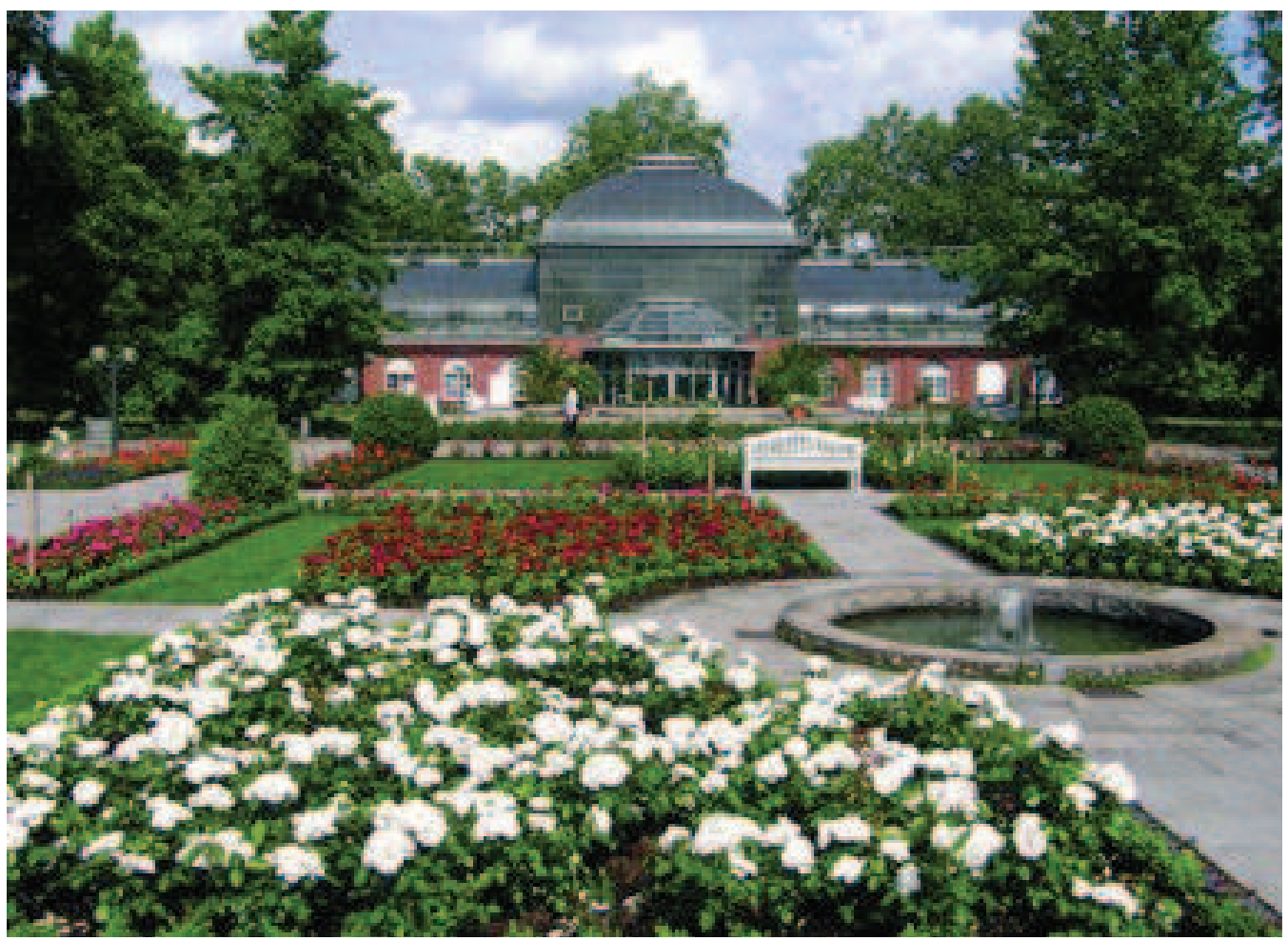

den entschieden, dass der Rindenmulch als Bodenbelag und Fallschutz ersetzt werden muss. Jetzt deckt Holzhäcksel den Boden um die Spielgeräte, da er Verletzungen besser vorbeugen kann.

Friseurtermin unter Palmen: Drei Athletinnen machen am 25. Mai im Palmengarten Reklame für die Olympia-Teilnahme von Eintracht Frankfurt. Hierzu kommen die drei Hammerwerferinnen BetTina HeIDlER, Kathrin KLAAS und ANDrEa Bunjes zu einem etwas außergewöhnlichen Friseurtermin in den Palmengarten. Außerdem wird erstmals die Ausstellung „Eintracht Frankfurt goes Bejing “ gezeigt. Ziel ist es, die Sportler den Bürgern der Region bekannt zu machen.

Seltener Stinker: Im Palmengarten blüht als größte Blume der Pflanzenwelt die Titanenwurz (Amorphophallus titanum), was immer wieder eine botanische Attraktion ist. Im Palmengarten öffnet sich die Blüte am 26. Juni und verbreitet nun ihren intensiven Aas-Gestank im Regenwaldhaus des Tropicariums. In ihrer Heimat Sumatra lockt die Pflanze so Aaskäfer an, die dann den Pollen verteilen.
Juli: Umweltdezernentin Dr. MANuela RotTMANN unterstützt den Palmengarten im Ausbau der Abteilung Betriebstechnik. Seit 20 Jahren wurde nichts mehr in diesen Bereich investiert, was $\mathrm{zu}$ einem regelrechten Investitionsstau führte. Ein Ziel der Modernisierung ist es, Energie- und Wasserverbrauch zu reduzieren.

Trauer im Festsaal: Für die Sanierung des Palmengarten-Gesellschaftshauses findet sich vorerst kein Generalunternehmer. Auch die Kosten werden neu berechnet. Sie bewegen sich jetzt mit 34,7 Millionen Euro rund 5,5 Millionen über der ersten Schätzung. Die Mehrkosten entstehen vor allem aus Gründen des Denkmalschutzes. Auch das Glasdach des Saales soll wiederhergestellt werden. Für den Palmengarten ist schwerwiegend, dass sich die Bauarbeiten verzögern. Wenn alles klappt, wird das Gesellschaftshaus 2011 fertig sein.

Abb. 6: Blick auf Eingangsschauhaus und den neu angelegten Rosengarten. 
Fotos unter Palmen: „Europäischer Naturfotograf des Jahres" heißt die Ausstellung, die vom 3. Juli bis 31. August in den Galerien des Palmenhauses die Fantasie beflügelt und dabei hochwertige Fotos vorstellt. Den Wettbewerb richtet die Gesellschaft Deutscher Tierfotografen jährlich aus und im Palmengarten werden 81 preisgekrönte Naturfotografien gezeigt. Beeindruckende Tierporträts und höchst ungewöhnliche Pflanzenbilder machen auf die Schönheit unserer Natur aufmerksam.

Gitarre, Schlagzeug, Bass: Den Startschuss für die Reihe "Jazz im Palmengarten“ der Jazzinitiative Frankfurt/Main e.V. liefert das multinationale Trio E_L_B mit Gitarrist NGUYÊN LÊ, Bassist Michel Benita und Drummer Peter ERsKINE. Aufgrund der Fußball-Europameisterschaft wird die Jazz-Reihe dieses Jahr später als in den Jahren zuvor eröffnet.

Verführung im Palmengarten: Die Kammeroper zeigt im Palmengarten JACQUES OFFENBACHs Operette „Die Großherzogin von Gerolstein". Die Premiere des kunterbunten und frechen Lustspiels um Macht und Machtmissbrauch am 19. Juli fällt buchstäblich ins Wasser. Es folgen für die Kammeroper aber noch etliche Abende mit deutlich besserem Wetter.

„Stadt genehmigt sich den Kahlschlag“, schreibt eine Zeitung. Vier Pinien, die zu den wenigen ihrer Art zählen, die nördlich der Alpen gedeihen, müssen aufgrund der anstehenden Sanierungsarbeiten des Gesellschaftshauses gefällt werden. Die Pinien des Palmengartens wurden in den 1980ern gepflanzt und gedeihen gut. Es ist daher sehr schade, dass die Bäume gefällt werden müssen. Einziger Trost ist, dass es am Oktogonbrunnen des Palmengartens noch weitere Pinien gibt.

Spendenkarussell im Palmengarten: Unter dem Motto „Kinder helfen Kindern“ stellt die Schaustellerfamilie FeUERSTEIN der HeINRICHKRAFT-Stiftung ein Karussell zur Verfügung. Der Palmengarten unterstützt diese Aktion mit einem geeigneten Platz. Jede Fahrt erhöht den

Abb. 7: Wasserfest: viel Spaß rund um das Wasser und Wasserpflanzen für die ganze Familie.

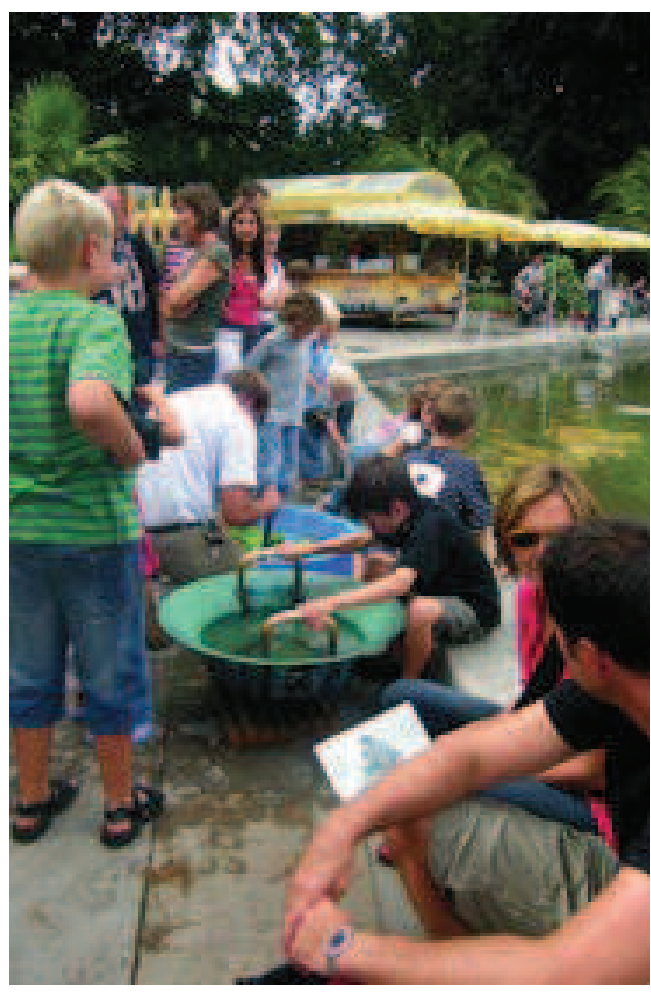

Spendenbetrag, mit dem die HeInRICH-KRAFTStiftung Kinder aus Frankfurter Heimen und Pflegefamilien unterstützt.

August: Die hohen Sommertemperaturen lassen uns Menschen schwitzen, doch einige Pflanzen gedeihen bei diesem Wetter besonders gut. Der Sommer lässt den Palmengarten als Märchen aus "Tausendundeiner Blume" in barocker Blütenund Farbenpracht erstrahlen. Es blüht und duftet zurzeit der Sommerflor mit seinen rund 40000 Pflanzen.

Gestalter des Grüns: MAX BROMME, der sogenannte „Vater der Frankfurter Parks“, feiert im Palmengarten seinen 90. Geburtstag. Er war von 1912 bis 1945 Gartenbaudirektor in Frankfurt und unter seiner Leitung entstanden viele bedeutende Grünflächen. So sind beispielsweise die Friedrich-EberT-Anlage und die Senckenberganlage während seiner Amtszeit entstanden. Er war außerdem für den Ausbau des Palmengartens verantwortlich.

Das GWH-Familienfest ist in diesem Jahr „wasserfest“. Rund um das Thema Wasser dre- 


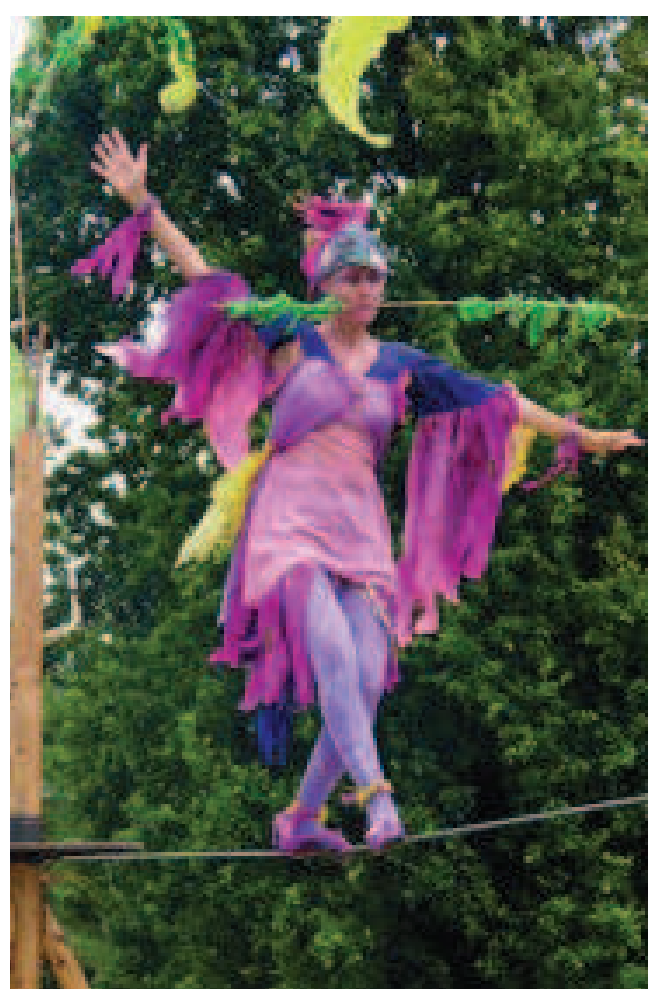

hen sich die Angebote an verschiedenen Plätzen des Palmengartens an diesem Tag. Die Grüne Schule bietet Bastelkurse für schwimmende Objekte an und in einem Experimentierzelt können Kinder Versuche zum Thema Wasser machen. Außerdem lassen die Seiltanzakrobaten „Luftgaukler" den Besuchern den Atem stocken.

Am 8.8.2008 geben sich im Palmengarten zehn Paare das Ja-Wort. „Für die Eheleute hat dieses Datum etwas Magisches, so wie eine Glückszahl“, sagt der Standesbeamte. Außerdem kann so der Hochzeitstag wohl kaum vergessen werden.

Das Papageno-Theater eröffnet am 9. August die neue Spielzeit mit einem „Kleinen Sommernachtstraum" für Kinder, einer märchenhaften Feengeschichte nach WILLIAM SHAKESPEARE mit Musik. Ab sofort findet somit wieder regelmäßig Kinderprogramm im Papageno statt.

GÜNTHER LeNZ gastiert mit Springtime im Palmengarten: Er ist einer der gefragtesten Bassisten und Komponisten der Jazzszene. Musikalisch bewegt er sich zwischen Neuer Musik und improvisiertem Jazz. Im Palmengarten tritt er im Rahmen von "Jazz im Palmengarten" zu- sammen mit Springtime auf und begeistert das Publikum.

Das kühle Wetter zum Jahresbeginn schlägt sich in den Besucherzahlen nieder. Im ersten Halbjahr 2008 kamen 366667 Besucher in den Palmengarten. Das sind $20 \%$ weniger als im Vorjahr.

Brasilien im Palmengarten: Im Rahmen einer Sonderausstellung wird im Tropicarium die artenreiche Pflanzenwelt des größten Landes Südamerikas vorgestellt. In keinem anderen Land der Erde gibt es so viele verschiedene Tier- und Pflanzenarten wie in Brasilien, insbesondere in Amazonien, dem weltweit größten zusammenhängenden Regenwaldgebiet. Auf Schautafeln der Uni Ulm werden Vegetation, Geschichte und Erforschung Brasiliens gezeigt.

September: Couture im Gewächshaus: Im September wird das Gewächshaus zum Laufsteg. Zwischen den exotischen Pflanzen und Blüten des Palmengartens präsentieren Mode-Designer aus dem Rhein-Main-Gebiet den Gästen, was in der kommenden Saison en vogue ist.

Zum diesjährigen Abschlusskonzert der Reihe „Weltmusik“ heizt das BOBAn I MARKOVIC Orkestar mit seinen Blechbläsern den Gästen ein. Nun kann der Herbst Einzug in den Palmengarten halten, denn die sommerlichen Konzertabende sind vorbei.

Besucher des Palmengartens fragen sich, wer da mitten im Brunnen vor dem Direktionsgebäude steht. Seit kurzem ist dort ein Mann mit leuchtend roten Haaren und einem Schimpansen auf der Schulter zu sehen. Die Installation trägt den Titel „HumbOLDTs Geist“ und stammt von dem kanadischen Künstler Trevor Gould. Eigens für den Palmengarten fertigte er diese Skulptur, die die Beziehung des Menschen zur Natur darstellen soll und bis Dezember zu sehen ist.

So viel Leben: Beim Wassertag im Palmengarten lernen Kinder spielerisch den Wert des Ele-

Abb. 8: Seiltanz-Vorführung während des GWH-Familienfestes. 
ments kennen. Der Verein „Umweltlernen in Frankfurt" lädt Schulkinder ein, um ihnen alles rund um das Thema Wasser, vom kleinsten Wassertropfen bis zu den großen Weltmeeren, zu erklären. Der Palmengarten stellt für den Wassertag die Spielwiese zur Verfügung, auf der unter anderem Regentropfen, Vogelgezwitscher oder Gewitter auf Instrumenten nachgespielt werden.

Der Palmengarten bekommt Verstärkung: Die 27-jährige Ingenieurin für Landschaftsbau Nicole HofmanN ist seit September Leiterin des Galerieteams und für die Ausstellungen zuständig. Gerade erst angekommen, organisiert sie bereits schon die aktuelle Erntedank-Schau und sorgt mit ihrem Team dafür, dass Sonnenblumen, Gladiolen, Astern und Dahlien dem Palmengarten einen goldenen Herbst bescheren, auch wenn es draußen trüb ist. Die Erntedankausstellung zeigt viele farbenfrohe Herbstblumen, Obst und Gemüse wie zum Beispiel Mais und Paprika. Auch ist der Herbst die Zeit der Kürbisse, die sich insbesondere beim Kürbisschnitzen während des Herbstfestes großer Beliebtheit erfreuen.

Oktober: Für Liebhaber ist sie schon eine Tradition, für Anfänger ein idealer Einstieg in die faszinierende Welt einer besonderen Pflanzengruppe - die Orchideenbörse im Palmengarten. Auch in diesem Jahr steht Altbewährtes, Außergewöhnliches und Neues aus der Welt der Orchideen im Mittelpunkt dieser Veranstaltung.

Japanische Gärten sind ,in“: Ein Vortrag über Japan-Gärten mit vielen Lichtbildern entführt die Besucher in die paradiesischen und zenbuddhistischen Gärten Japans, gibt viele Einblicke und Hintergrundinformationen und macht Lust auf einen Japan-Garten.

Edle Pflanze Chu: Für eine Woche stellt sich der Palmengarten ganz auf Fernost ein. Chrysanthemen, die zu den vier edlen Pflanzen des alten Chinas zählen und dort als „Chu“ bekannt sind, werden in den Galerien ausgestellt. Dazu gibt es

Abb. 9 (oben): Fleißiges Eichhörnchen im Palmengarten. Abb. 10 (unten): Chrysanthemen-Ausstellung.
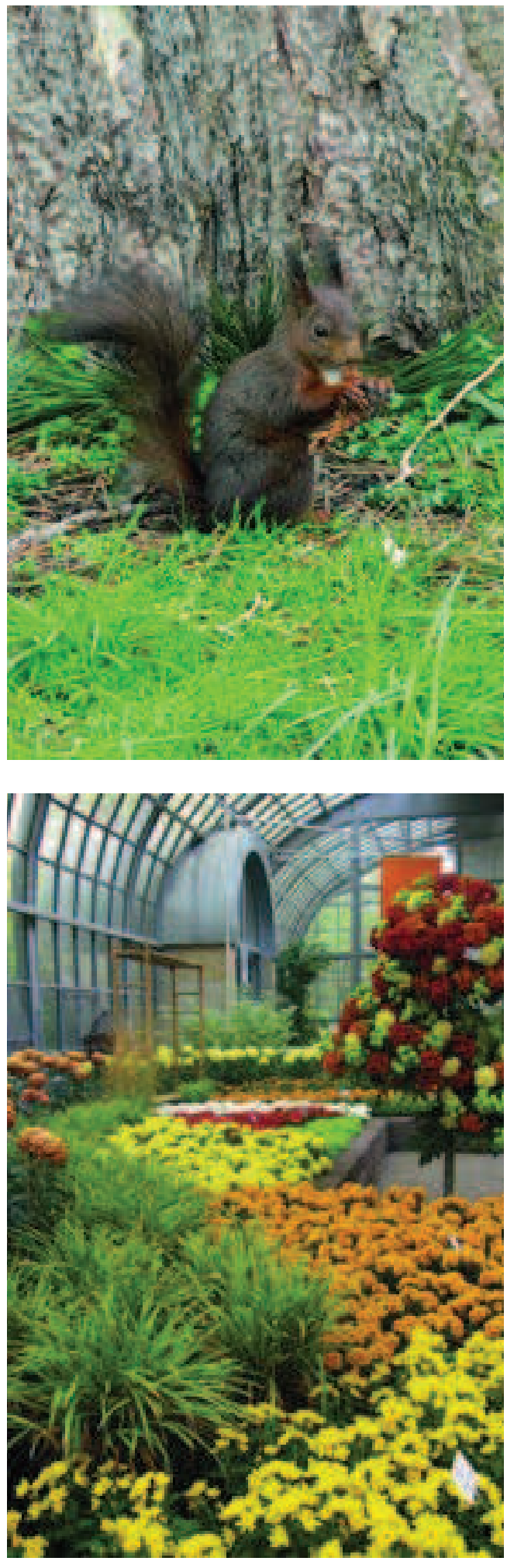


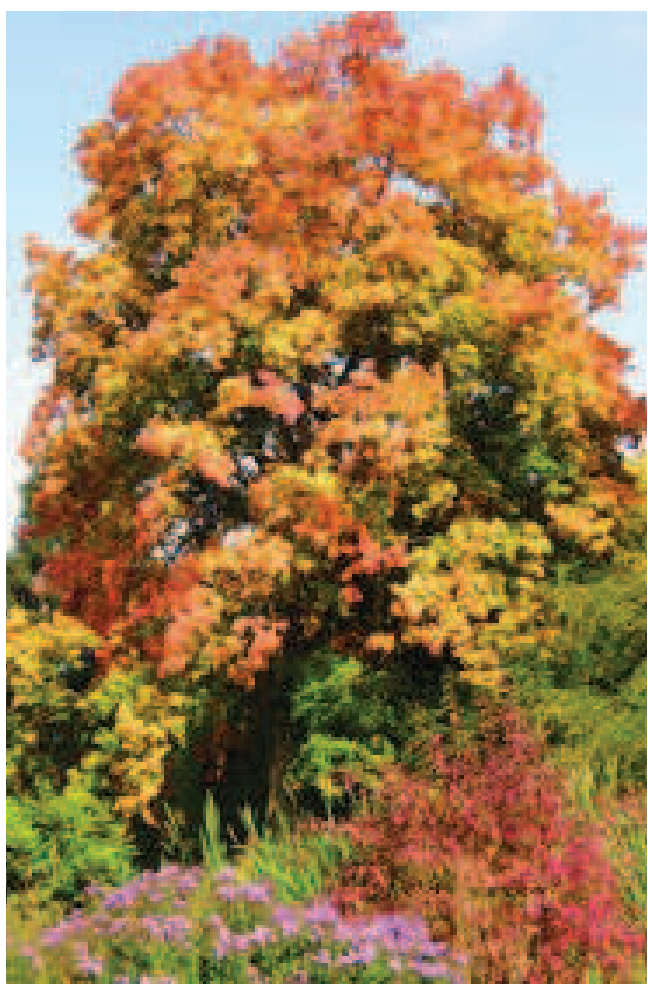

Teezeremonien und Vorführungen der kalligraphischen Kunst. Wer sich nach einem langen Spaziergang oder Besuch der Ausstellung noch etwas Gutes tun möchte, kann sich im Palmengarten sogar nach chinesischer Tradition massieren lassen.

Goldener Oktober: Das Hoch „Hagen“ beschert uns einen schönen, goldenen Oktober, und somit ist die Herbstfärbung im Garten dieses Jahr besonders schön. Neben all den bunten Herbstblättern schießen außerdem überall Pilze aus dem Boden.

Der Oktober ist auch der Monat der Frankfurter Buchmesse. Der Palmengarten ist der ideale Erholungsort für die Messeteilnehmer. So startet die in Italien lebende Buchautorin PeTRA RESKI gerne ihren Messetag im Palmengarten: „Zum Frühstücken finde ich das Café SIESMAYER im Palmengarten sehr schön. So einen altmodisch botanischen Garten hat ja nicht jede Stadt."

Hoch und Tief: Neuerdings kommen die Daten für den Wetterdienst nicht mehr aus dem Palmengarten, sondern von einer Wetterstation der GoETHE-Universität. Auf dem Campus Westend hat der Wetterdienst seine neue, vollautomatische Online-Station in Betrieb genommen, nachdem die Messungen aus dem Palmengarten wegen zunehmender Verschattung durch die Bäume nicht mehr internationalem Standard genügten. Nun werden mit Hilfe modernster Geräte kontinuierlich die Niederschlagsmenge, Luft- und Bodentemperaturen sowie Luftdruck an die Zentrale des Wetterdienstes nach Offenbach übermittelt.

Schauspieler SIEMEN RÜHAAK, derzeit im FRITZ-RÉMOND-Theater zu sehen, zeigt seinem Besuch gerne besondere Orte in Frankfurt. Seine Wege führen auch in den Palmengarten. „Ich habe meine Texte früher immer im Palmengarten gelernt und meine Tochter ist im Garten rumstolziert", erinnert sich RÜHAAK gern an alte Zeiten.

November: Studenten der European School of Design haben sich Gedanken über ein Leitsystem zur besseren Besucherorientierung gemacht. Das gibt es im Palmengarten noch nicht, somit sind dem Ideenreichtum der jungen Designer keine Grenzen für ihre Arbeit gesetzt. Für die Studenten erweist sich der Palmengarten als „schier unerschöpfliches Betätigungsfeld“, so die Dozentin der Schule. Die grafischen Arbeiten werden bis zum 23. November ausgestellt.

Zum Geburtstag einen Wasserfall: Der „kreative Verschönerer" und Edel-Florist ERHARD PRIEWE feiert im Palmengarten seinen 50. Geburtstag. Rund 200 Freunde und Weggefährten kommen zur Feier in den von ihm dekorierten Garten. PRIEWE wünscht sich zum Geburtstag von seinen Gästen keine Geschenke, stattdessen eine Spende für einen Wasserfall im Tropicarium.

Stadtplanung im Klimawandel: Im Rahmen der Veranstaltungsreihe „Frankfurt bleib(t) cool“ lädt das Umweltdezernat zu der Informationsveranstaltung „Stadtplanung im Klimawandel“ in den Palmengarten. Besonders große Heraus-

Abb. 11: Prächtige Herbstfärbung. 
forderungen für Stadtplaner und Architekten der Zukunft stellen Themen wie Hitzewellen, Überschwemmungen und tropische Temperaturen in den Städten dar.

Eine von den Vereinten Nationen (UN) initiierte Foto-Ausstellung "Chasing the Dream" wird im Palmengarten eröffnet. Es werden acht Porträts von Jugendlichen aus der ganzen Welt gezeigt. Mit seiner jeweiligen Geschichte steht jedes dieser Porträts für eines der acht UN-Entwicklungsziele (z. B. Schulbildung, Bekämpfung von HIV und Hunger), die im Jahre 2000 als Millenniumsziele beschlossen wurden und bis 2015 verwirklicht werden sollen. Die Globalisierung verbindet das Alltagsleben in Deutschland mit dem in der ganzen Welt. Durch die Ausstellung sollen die Besucher angeregt werden, über diese Verbindung und ihr eigenes Handeln nachzudenken.

Der neue Reiher vom Palmengarten: Majestätisch und mit erhobenem Kopf steht der neue Graureiher da und hat sein frisch bezogenes Revier im Blick. Fischzüchter sehen die Tiere ungern, im Palmengarten jedoch freuen sich die Besucher am Anblick des Vogels, der oft auf der Weide am Bootsweiher sitzt oder elegant über den Weiher fliegt. Dieser Graureiher ist jedoch nicht, wie viele denken, derjenige, den sie schon seit Jahren aus dem Palmengarten kennen. Der ist leider schon vor einiger Zeit verstorben. „Die Natur richtet es so ein, dass Nischen schnell geschlossen werden - jetzt ist der Neue da", freut sich der Tierbeauftragte HEINZ SCHAAN.

Dezember: Auf die Köstlichkeiten der Weihnachtszeit kann man sich im Palmengarten sinnlich einstimmen: Die Grüne Schule stellt tropische Weihnachtsgewürze vor und erläutert, warum Zimt und Nelken, Vanille, Safran und Kardamom seit alters her so begehrt sind.

Abb. 12: HuMBOLDT hat sich über Nacht in einen NiKOLAUS verwandelt.

Abb. 13 (S. 12, oben): Der Winter sorgt für Formenspiele: Granatapfelfrucht-Vogel mit Schneehäubchen.

Abb. 14 (S. 12, unten): Weihnachten im Palmengarten.

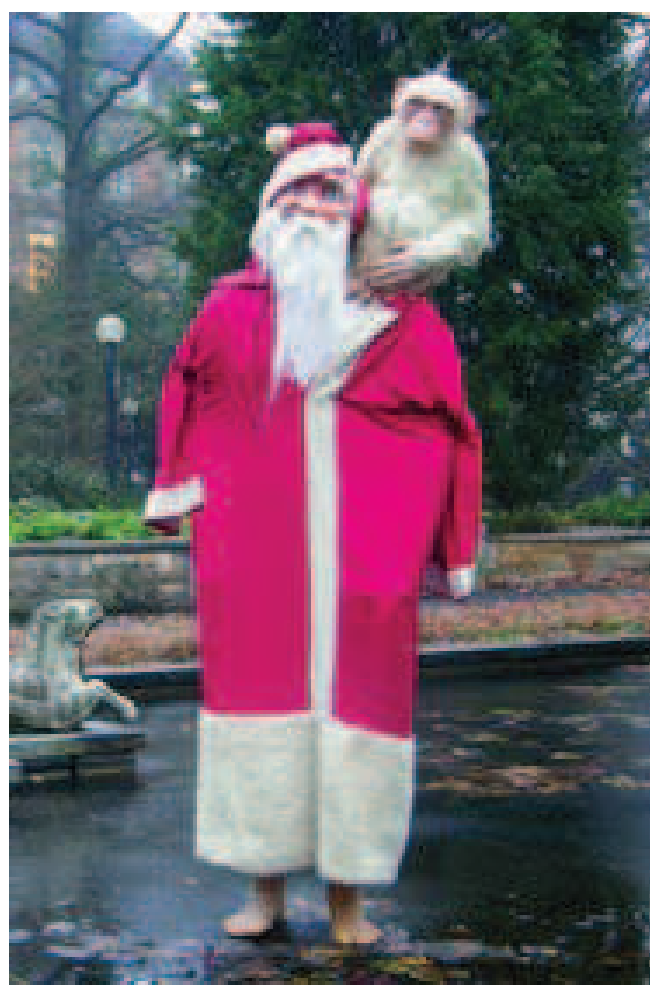

Palmengarten unterstützt LEBERECHT-Stiftung: In der Fressgass sammeln engagierte Helfer, darunter natürlich auch der Palmengartendirektor, rund 20000 Euro für die Stiftung LeBERECht. Unter anderem werden bei dieser Aktion Alpenveilchen verkauft, die vom Palmengarten zur Verfügung gestellt werden. Der Erlös kommt behinderten und benachteiligten Kindern zugute.

Mit rund 170 Gästen kommen dieses Jahr bereits zur Eröffnung der Ausstellung „Blüten und Gehölze zur Weihnachtszeit" besonders viele Besucher zur etwas anderen Weihnachtsausstellung. Es werden traditionelle und moderne weihnachtliche Dekorationsideen gezeigt. Es gibt Rezepte für ungewöhnliche Plätzchensorten wie „Wüstensand“ oder „Schokosalami“, und wer sein Zuhause noch nicht ausreichend geschmückt hat, kann sich zahlreiche Anregungen holen oder gleich selbst seinen Weihnachtsschmuck im Palmengarten basteln.

Ein NiKOLAus im Palmengartenteich: „HuMBOLDTs Geist“, die Skulptur des kanadischen Künstlers TRevor Gould, die seit September im Teich vor dem Verwaltungsgebäude steht, 


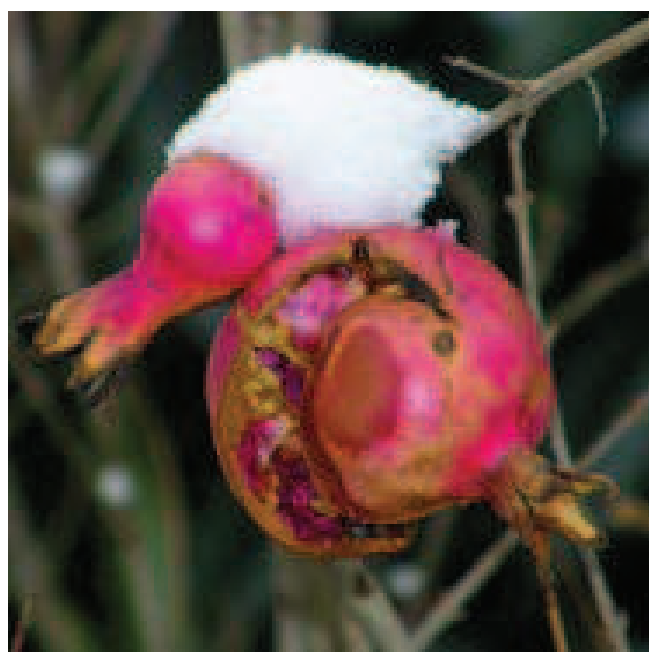

wurde Opfer einer Nacht- und Nebel-Aktion. Die Figur bekam einen Nikolausmantel, eine Maske und rote Zipfelmütze verpasst. Unbe- kannt ist, wer diese eher unkünstlerische Tat verübt hat.

Das Papageno-Musiktheater veranstaltet ein weihnachtliches Benefizkonzert mit vielen Nachwuchs-Opernkünstlern. Das Konzert unterstützt eine künftige Produktion, das Musical „Der Mann von La Mancha“.

Palmengarten-Führer aktualisiert: Im Palmengarten gab es in den vergangenen Jahren manche Neuerung wie die Sanierung des Rosengartens oder das Umbauprojekt am historischen Gesellschaftshaus. Deshalb ist nun auch der handliche Palmengarten-Führer in einer aktualisierten Auflage erschienen. Hierfür hat die Autorin Beate TAudte-Repp alle Angaben auf den neuesten Stand gebracht und zusätzliche Fotos eingebaut. Das 144 Seiten umfassende Büchlein ist an den Kassen sowie in der Boutique des Palmengartens erhältlich.

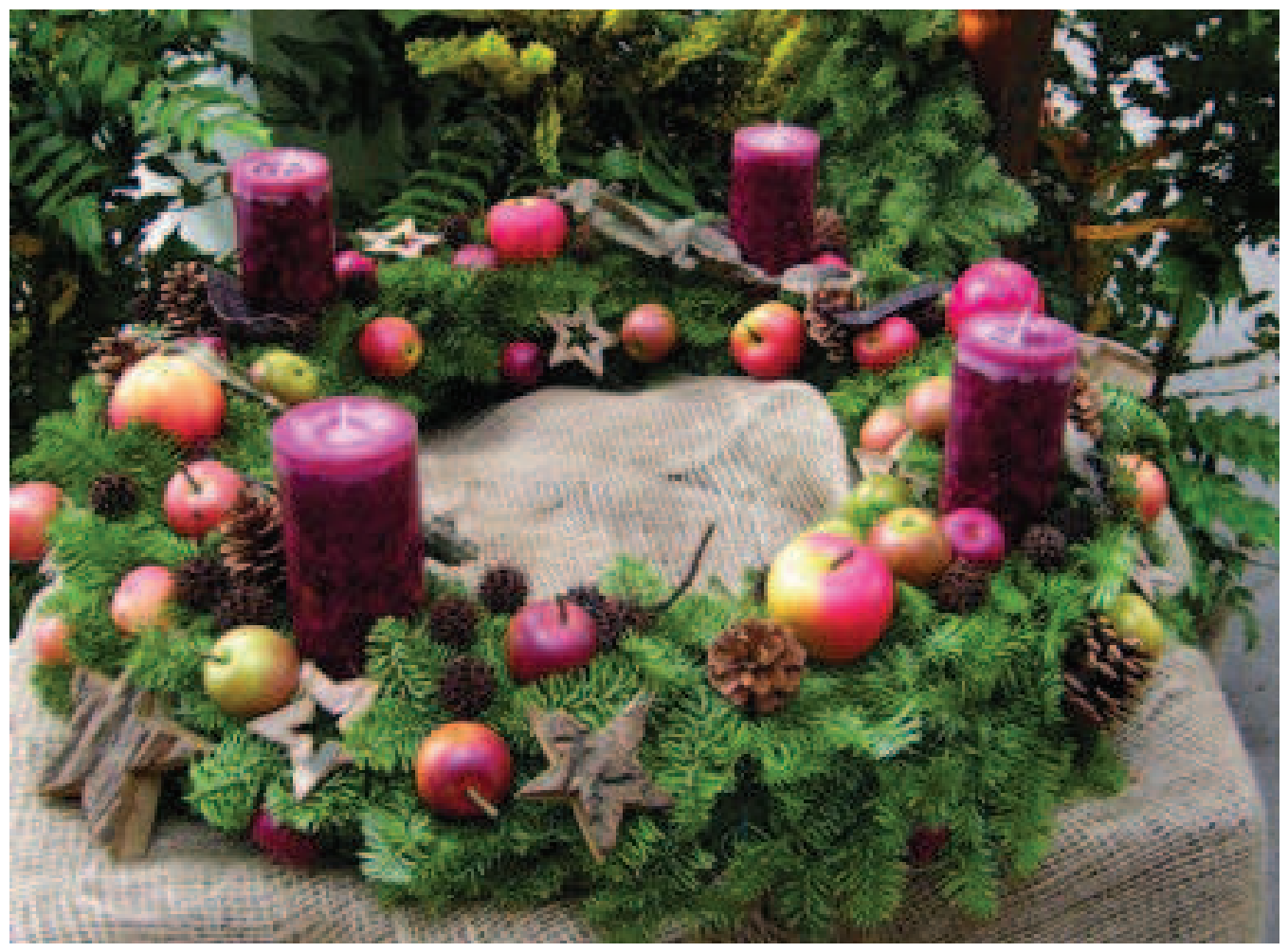

